\title{
Gastroenterologist and nurse management of symptoms after pelvic radiotherapy for cancer: an economic evaluation of a clinical randomized controlled trial (the ORBIT study)
}

\author{
This article was published in the following Dove Press journal: \\ ClinicoEconomics and Outcomes Research \\ 28 April 2017 \\ Number of times this article has been viewed
}

\author{
Jake Jordan' \\ Heather Gage' \\ Barbara Benton ${ }^{2}$ \\ Amyn Lalji² \\ Christine Norton ${ }^{3}$ \\ $\mathrm{H}$ Jervoise $N$ Andreyev ${ }^{2}$ \\ 'Surrey Health Economics Centre, \\ School of Economics, University of \\ Surrey, Guildford, ${ }^{2}$ Gastrointestinal \\ Unit, Royal Marsden NHS Foundation \\ Trust, ${ }^{3}$ Florence Nightingale Faculty of \\ Nursing and Midwifery, King's College, \\ London, UK
}

\begin{abstract}
Background: Over 20 distressing gastrointestinal symptoms affect many patients after pelvic radiotherapy, but in the United Kingdom few are referred for assessment. Algorithmic-based treatment delivered by either a consultant gastroenterologist or a clinical nurse specialist has been shown in a randomized trial to be statistically and clinically more effective than provision of a self-help booklet. In this study, we assessed cost-effectiveness.

Methods: Outcomes were measured at baseline (pre-randomization) and 6 months. Change in quality-adjusted life years (QALYs) was the primary outcome for the economic evaluation; a secondary analysis used change in the bowel subset score of the modified Inflammatory Bowel Disease Questionnaire (IBDQ-B). Intervention costs, British pounds 2013, covered visits with the gastroenterologist or nurse, investigations, medications and treatments. Incremental outcomes and incremental costs were estimated simultaneously using multivariate linear regression. Uncertainty was handled non-parametrically using bootstrap with replacement.
\end{abstract}

Results: The mean (SD) cost of treatment was $£ 895$ (499) for the nurse and £1101 (567) for the consultant. The nurse was dominated by usual care, which was cheaper and achieved better outcomes. The mean cost per QALY gained from the consultant, compared to usual care, was $£ 250,455$; comparing the consultant to the nurse, it was $£ 25,875$. Algorithmic care produced better outcomes compared to the booklet only, as reflected in the IBDQ-B results, at a cost of $\sim £ 1,000$. Conclusion: Algorithmic treatment of radiation bowel injury by a consultant or a nurse results in significant symptom relief for patients but was not found to be cost-effective according to the National Institute for Health and Care Excellence (NICE) criteria.

Keywords: pelvic radiotherapy, algorithmic care, gastroenterologist, clinical nurse specialist

\section{Introduction}

Up to 23 distressing gastrointestinal symptoms, including fecal incontinence, defecatory urgency, loose stools and abdominal pain, have been described to affect up to half of all patients who have undergone radiotherapy to treat a cancer in the pelvis. ${ }^{1,2}$ In the UK, however, only a small minority of survivors are ever referred for treatment for these symptoms, despite the serious repercussions they have on their quality of life..$^{3,4}$ Although previously considered refractory, a three-arm clinical randomized controlled trial (RCT) (ORBIT), completed in 2012, has shown that such chronic symptoms of radiation-induced bowel injury can be improved by algorithm-based treatment led by either a gastroenterologist or a clinical nurse specialist. ${ }^{5}$ The primary end point in this study was the bowel subset score of the Modified Inflammatory Bowel Disease
Correspondence: Heather Gage Surrey Health Economics Centre, School of Economics, University of Surrey, Stag Hill, Guildford GU2 7XH, UK

Email h.gage@surrey.ac.uk

$\mathrm{Tel}+441483686219$ 
Questionnaire (IBDQ-B). ${ }^{6,7}$ A statistically and clinically significant improvement in IBDQ-B was recorded for both the gastroenterologist group and the nurse group, when compared to a control group who were given a self-help booklet. ${ }^{5}$ The step-by-step algorithm was designed prior to the trial through a series of pilot studies that tested investigations and sequential treatments for the 23 symptoms that develop after radiotherapy. ${ }^{8-13}$ Application of the algorithm means that patients receive tests and treatments that are tailored to their individual needs.

Because of rising healthcare costs and limited budgets, new treatments are increasingly required to demonstrate valuefor-money as well as clinical efficacy. This article reports the economic evaluation of the ORBIT trial. The costs of implementing the algorithm by both the gastroenterologist and the nurse, and the findings from a cost-effectiveness analysis, are presented. Each professional is compared with the control (booklet) condition, and with each other. The primary analysis was a cost-utility analysis where the effectiveness metric, quality-adjusted life years (QALYs), reflects morbidity or health-related quality of life associated with the alternative strategies. QALYs provide a common unit of measurement through which the value of interventions in different areas can be compared. ${ }^{14}$ The National Institute for Health and Care Excellence (NICE), which provides guidance and quality standards for health, public health and social care in the UK, uses a threshold cost per QALY gained of between $£ 20,000$ and $£ 30,000$ to determine the cost-effectiveness in health technology appraisals. ${ }^{15}$ The secondary analysis concerned the cost per unit of therapeutic gain from the algorithm, using the IBDQ-B.

\section{Methods}

\section{Participants}

The ORBIT trial was conducted at the Royal Marsden Hospital in London between 2007 and 2011. Full details have been published elsewhere. ${ }^{5}$ Participants were aged 18 years or older, with persisting bowel symptoms that had started either during or following pelvic radiotherapy given curatively for various urological, gynecological and gastrointestinal cancers. They had to be well enough to travel to and from the hospital and be treated as outpatients. To be eligible, radiotherapy must have completed at least 6 months prior to enrollment in the study. Patients were excluded if they had serious comorbidities, metastatic disease, short life expectancy ( $<1$ year), a colostomy or ileostomy (unless reversed more than 6 months previously) or been assessed previously by a gastroenterologist for the symptoms. Patients were withdrawn from the study if they required treatment for recurrence of cancer or were hospitalized for the treatment of gastrointestinal symptoms during the study.

\section{Recruitment and data collection}

Potentially eligible patients were identified through the hospital radiotherapy department. Clinical records were searched for current or past patients meeting the eligibility criteria. Invitations to participate were delivered either by direct contact at normal patient follow-up appointments, or by telephone and/or mail. Patients referred to a routine National Health Service (NHS) outpatient clinic specializing in post-radiotherapy bowel symptoms for non-urgent treatment were also invited to take part.

All volunteers were screened for eligibility and invited to attend an outpatient clinic to complete consenting processes and provide baseline information. Participants were then randomized (block randomization stratified by tumor site and degree of bowel dysfunction, that is, IBDQ-B $<60$ or $\geq 60$ ) to one of three treatment groups: usual care (consisting of a self-help booklet), specialist nurse-led algorithmically driven care and consultant gastroenterologist-led algorithmically driven care. The trial duration was 1 year. Data were collected at baseline, 6-month and 12-month follow-up. At the 6-month follow-up, patients in the usual care (booklet) arm were given the option to transfer to the consultant arm if they had persisting gastrointestinal symptoms. For this reason, the analysis was conducted at the 6-month time point.

\section{Health outcome measures}

The primary outcome for the economic evaluation was a participant's QALY, obtained from Medical Outcomes Study's Short Form 12 health survey (SF-12) scores that were gathered at each assessment point. SF-12 is a well-validated and widely used generic measure of health-related quality of life that incorporates general health, mental health, pain, vitality, and physical, social and emotional functioning across 12 items. ${ }^{16}$ Responses can be converted to a reduced six dimension score (the SF-6D), which can be used to estimate single health utility index scores ranging from 0 (death) to 1 (perfect health) using standardized utility weights. ${ }^{17}$ These utility scores were then mapped to EQ-5D (EuroQoL) using a published procedure. ${ }^{18,19} \mathrm{EQ}-5 \mathrm{D}$ is the widely used generic instrument, with a UK utility tariff, ${ }^{20}$ which is favored by NICE to estimate health utility index scores. Using EQ-5D mapped utility estimates allows direct comparability of the cost-effectiveness results to other studies conducted using the EQ-5D to elicit QALY values. To provide an estimate of a participant's QALYs accrued over the 6-month time period, the area under the curve between baseline and 6 months was 
calculated using the trapezoid method (also known as discrete time integration).

Secondary analysis was conducted using the IBDQ-B change from baseline (the primary outcome for the clinical study), ${ }^{5}$ in place of the patient's QALY. Being a conditionspecific measure of health-related quality of life with scores ranging from 10 (worst possible bowel function) to 70 (perfect), the IBDQ-B is more sensitive to small changes in patient outcomes that may not be detected by the generic EQ-5D scale. A shift of six or more points in the IBDQ-B is considered to be a clinically relevant change from the patient perspective. $^{21}$

\section{Costs}

The cost of the intervention is made up of several elements: the number and duration of visits with either the consultant gastroenterologist or the clinical nurse specialist, and the cost of any investigations and treatments delivered as part of the algorithm, including medications. All costs were calculated at the individual patient level in British pounds, 2013, and measured from the perspective of the National Health Service.

The intervention consisted of an initial meeting with the consultant or the nurse, at which the patients' symptoms were assessed and tests and treatments were ordered according to the algorithm. Additional follow-up visits with the consultant or the nurse were arranged, as required. Unit costs for clinical contact were fully absorbed, inclusive of oncosts and overheads, and obtained from NHS reference costs, ${ }^{22}$ or Department of Health sources. ${ }^{23}$ The duration of the initial meeting was recorded in minutes, and the cost was calculated pro rata. For any subsequent outpatient appointments, the duration is not known so these follow-up sessions were assumed to have a 15-minute duration for both the consultantled and nurse-led intervention arms. The investigations and treatments delivered to patients were retrieved from patient notes at the end of the study, and costs were provided by the hospital finance department. All cost elements were summed to provide a total cost per patient of the intervention over the first 6 months.

\section{Analysis}

All economic analyses were conducted using STATA13 (Stata Statistical Software, release 13; StataCorp LP, College Station, TX, USA). Results presented are based on a complete case analysis for those subjects with complete data at baseline and 6 months, covering resource use, IBDQ-B and SF-12. Three sets of analysis were conducted: nurseled intervention was compared with usual care (booklet), consultant-led intervention was compared with usual care and, finally, consultant-led care was compared directly with nurse-led care.

\section{Cost utility (QALY)}

The cost-effectiveness of the intervention was assessed as an incremental cost-effectiveness ratio (ICER). This is the incremental cost per QALY gained, when moving from usual care (booklet arm) to either nurse-led algorithmic care, consultant-led algorithmic care or comparing nurse with consultant.

The incremental costs and incremental QALYs were estimated simultaneously using a seemingly unrelated regression (SUR). No covariates were included for estimation of costs. However, in the QALY equation, baseline utility score was included to control for patient-level differences at baseline. ${ }^{24}$

Uncertainty was handled non-parametrically using bootstrap with replacement. ${ }^{25}$ Samples $(n=10,000)$ of equal size to the original sample were drawn, and the incremental costs and QALYs were estimated using the SUR for each of the bootstrap samples. The results of this process are presented on a cost-effectiveness plane. In addition, the probability that each treatment would be deemed cost-effective at the NICE threshold willingness to pay of $£ 20,000-£ 30,000$ per QALY was calculated and presented in a cost-effectiveness acceptability curve and cost-effectiveness acceptability frontier (CEAF).

\section{Cost-effectiveness (IBDQ-B)}

The same non-parametric bootstrap sampling approach was used, as described earlier, using the IBDQ-B outcomes, and the results were presented as cost per six-point (clinically relevant) change in IBDQ-B score.

\section{Ethical considerations}

The ORBIT study gained a favorable ethical opinion following review by the Royal Marsden Research Ethics Committee (later named NRES Committee London-Chelsea), reference 07/Q0801/47. All participants provided informed written consent for their data to be used in the study. The trial is registered with ClinicalTrials.gov, number NCT00737230.

\section{Results}

\section{Characteristics of participants}

Of 2,484 patients assessed for eligibility, 218 participated and the rest were ineligible ( $\mathrm{n}=876$ ), not contactable (185), reported no new symptoms (934) or refused (271). ${ }^{5}$ At randomization, 68, 80 and 70 participants were allocated to the usual care (booklet), nurse-led algorithmic care and consultant-led algorithmic care groups, respectively. At 6 months, loss to follow-up and withdrawals from the study reduced 
these sample sizes to 57,62 and 59 , respectively $(n=178)$. Of these 178 participants, $159(47,61,51)$ had complete data on the SF-12 at baseline and 6 months, sufficient to estimate a health utility index. Three of the 159 patients had no information captured on the duration of their baseline clinical consultation, so simple imputation was applied using the mean duration for the appropriate treatment arm, rather than dropping the patients from the analysis. There were 182 (57, $66,59)$ patients with IBDQ-B data at baseline and 6 months.

\section{Costs}

Based on those patients with complete data for costs and SF-12 at baseline and 6 months, the unconditional mean (SD) cost of treatment was $£ 895$ (499) and $£ 1101$ (567) for nurse and consultant-led treatments, respectively (Table 1). The consultant was more costly than the nurse in clinical time (due to higher unit costs) and in use of investigations and prescribing. The higher value of medications in the consultant arm is driven by a few outliers (median [interquartile range] medication cost $£ 1.05$ (0-35) for nurse and $£ 25.80(0-35)$ for consultant). When the complete case sample was delimited based on IBDQ$B$ rather than SF-12, the sample size increased marginally, but this did not result in a significant change in resource use or costs (Table 1). The cost of treatment for the usual care arm (booklet) is treated as zero, since there is no required clinical contact, or additional prescription of medication.

\section{Outcomes}

All three treatment arms saw small improvements in utility between baseline and 6-month follow-up, the smallest mean increase being in the nurse-led arm. None of the changes were statistically significantly different from zero. The mean QALY gains over the 6-month period in the consultant and nurse arms were not significantly different from those in the booklet arm, and there was no significant difference between the improvements in the nurse or consultant groups (Table 2).

The mean IBDQ-B scores improved in all three groups over the 6 months. The change in the mean IBDQ-B score was larger in the consultant than nurse arm, but the difference between these two changes was not statistically significant. However, the changes in both intervention arms were significantly larger than the change in the booklet arm.

\section{Cost-utility analysis}

In the cost-utility analysis (Table 3; Figure 1), the nurse-led arm was dominated by the usual care (booklet arm), being more expensive and having worse health (QALY) outcomes in approximately two thirds of the 10,000 sampling realizations. The consultant-led treatment, compared to usual care, was more expensive but led to better health outcomes in more than half of the sampling realizations (mean cost per QALY gained $[$ ICER] $=£ 250,455)$. When the consultant-led treatment is compared to the nurse-led treatment, there is a small incremental cost, but in the majority of sampling realizations, the consultant-led treatment resulted in better health outcomes (mean cost per QALY gained [ICER] $=£ 25,875$ ).

For each of the 10,000 realizations, the three treatments can be ranked in terms of which yielded the most cost-effective result for a given willingness to pay per QALY. The frequency of 'wins' from these rankings over the 10,000 samples is an estimate of the probability the treatment would be deemed the most cost-effective when compared to the alternatives. This analysis generates the CEAF. The nurse-led treatment option is dominated by both the booklet and consultant-led treatment options. At the lower willingness to pay per QALY, the booklet is favored and, at a WTP of $£ 30,000$ (the upper limit normally imposed by NICE), the booklet arm is predicted to have a near $100 \%$ likelihood of being the most cost-effective. Above

Table I Resources and costs (British pounds 20I3)

\begin{tabular}{|c|c|c|c|c|c|c|c|c|c|c|c|c|c|}
\hline \multirow[t]{2}{*}{$\begin{array}{l}\text { Treatment } \\
\text { group }\end{array}$} & \multirow[t]{2}{*}{$\mathbf{N}$} & \multicolumn{3}{|c|}{ Baseline consultation duration } & \multicolumn{3}{|c|}{ Additional consultations $^{\mathrm{a}}$} & \multicolumn{2}{|c|}{$\begin{array}{l}\text { Investigations, } \\
\text { treatments }\end{array}$} & \multicolumn{2}{|c|}{ Medications } & \multicolumn{2}{|l|}{ Total cost } \\
\hline & & Mean (min) & SD (min) & $\operatorname{Cost}^{\mathrm{b}}(t)$ & Mean, $\mathbf{n}$ & SD & $\operatorname{Cost}^{\mathrm{b}}(t)$ & $\begin{array}{l}\text { Mean } \\
\operatorname{cost}^{c}(t)\end{array}$ & SD & $\begin{array}{l}\text { Mean } \\
\cos ^{\mathrm{d}}(t)\end{array}$ & SD & Mean $(t)$ & SD \\
\hline \multicolumn{14}{|c|}{ Based on participants with SF-12 (QALY) data at baseline and 6 months } \\
\hline Nurse & 61 & 47.5 & 21.5 & 70 & 1.03 & 0.86 & 22.66 & 786 & 493 & 17 & 23.77 & 895 & 499 \\
\hline Consultant & 51 & 29.7 & 15.8 & 162 & 0.98 & 0.95 & 80.12 & 793 & 527 & 66 & 152.46 & 1101 & 567 \\
\hline \multicolumn{14}{|c|}{ Based on participants with IBDQ-B data at baseline and 6 months } \\
\hline Nurse & 66 & 47.8 & 20.9 & 70. & 1.03 & 0.86 & 22.66 & 768 & 494 & 17 & 23.25 & 878 & 498 \\
\hline Consultant & 59 & 29.4 & 14.9 & 159 & I & I & 82.60 & 764 & 536 & 69 & 166.7 & 1075 & 580 \\
\hline
\end{tabular}

Notes: a Duration based on 15 -minute consultation. bUnit costs for specialist nurse is $£ 88 /$ hour ${ }^{23}$ and for gastroenterologist is $£ 162 /$ consultation of 30 minutes. ${ }^{22}$ C Costs of investigations and treatments supplied by the hospital finance department: abdominal X-ray $£ 49.95$; anal ultrasound $£ 77.23$; bloods battery $£ 75$. I2; colonoscopy $£ 683$.00; $C T$

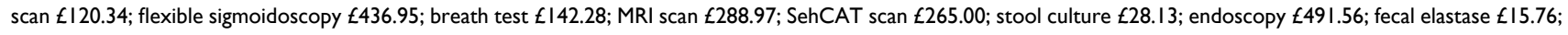
liver ultrasound $€ 77.23$. 'Total cost of medications per patient supplied by clinical team.

Abbreviations: CT, computerised tomography; IBDQ-B, Inflammatory Bowel Disease Questionnaire; MRI, magnetic resonance imaging; QALY, quality-adjusted life year; SehCAT scan, selenium homocholic acid taurine scan; SD, standard deviation; SF-12, Medical Outcomes Study's Short-Form 12. 
Table 2 Outcomes

\begin{tabular}{|c|c|c|c|c|c|c|c|}
\hline \multirow[t]{2}{*}{ Treatment group } & \multirow[t]{2}{*}{$\mathbf{N}$} & \multicolumn{2}{|c|}{$\begin{array}{l}\text { Utility score } \\
\text { (baseline) }\end{array}$} & \multicolumn{2}{|c|}{$\begin{array}{l}\text { Utility score } \\
\text { (6 months) }\end{array}$} & \multicolumn{2}{|c|}{$\begin{array}{l}\text { Incremental QALYs } \\
\text { gained versus booklet } \\
\text { group }^{\mathbf{a}}\end{array}$} \\
\hline & & Utility & SD & Utility & SD & Mean & SD \\
\hline \multicolumn{8}{|c|}{ Based on participants with SF-I2 (QALY) data at baseline and 6 months } \\
\hline Booklet & 47 & 0.776 & 0.200 & 0.797 & 0.170 & & \\
\hline Nurse & 61 & 0.789 & 0.172 & 0.792 & 0.177 & -0.0036 & 0.005 \\
\hline Consultant & 51 & 0.799 & 0.165 & 0.829 & 0.157 & 0.0044 & 0.005 \\
\hline \multirow[t]{2}{*}{ Consultant vs. nurse } & 51 & & & & & 0.0076 & 0.006 \\
\hline & $\mathbf{N}$ & $\begin{array}{l}\text { IBDQ-I } \\
\text { (baselir }\end{array}$ & & $\begin{array}{l}\text { IBDQ- } \\
\text { (6 mon }\end{array}$ & & \multicolumn{2}{|c|}{$\begin{array}{l}\text { Incremental change } \\
\text { in IBDQ-B score vs. } \\
\text { booklet group }^{\mathrm{a}}\end{array}$} \\
\hline \multicolumn{8}{|c|}{ Based on participants with IBDQ-B data at baseline and 6 months } \\
\hline Booklet & 57 & 52.53 & 12.66 & 57.46 & 12.86 & & \\
\hline Nurse & 66 & 52.95 & 10.13 & 62.00 & 10.21 & $4.12^{\mathrm{b}}$ & 2.0 (SE) \\
\hline Consultant & 59 & 52.62 & 10.93 & 60.68 & 10.76 & $5.07^{\mathrm{b}}$ & $2.16(\mathrm{SE})$ \\
\hline Consultant vs. nurse & & & & & & 0.95 & $\mathrm{I} .68(\mathrm{SE})$ \\
\hline
\end{tabular}

Notes: alncremental QALY gain based on ordinary least squares regression estimates controlling for baseline utility. ${ }^{\text {b }}$ ifferences between groups statistically significant at a $5 \%$ level using two-sample t-test. Within-group changes were significant for nurse and consultant, but not for booklet. ${ }^{5}$

Abbreviations: IBDQ-B, Inflammatory Bowel Disease Questionnaire; SD, standard deviation; QALY, quality-adjusted life year; SE, standard error; SF-12, Medical Outcomes Study's Short-Form 12.

Table 3 Cost-utility and cost-effectiveness analysis

\begin{tabular}{|c|c|c|c|c|}
\hline Cost-utility analysis & $\mathbf{N}$ & Incremental cost $(t)$ & $\begin{array}{l}\text { Incremental mean change } \\
\text { in QALY }\end{array}$ & $\begin{array}{l}\text { ICER (incremental cost } \\
\text { per QALY gained) }\end{array}$ \\
\hline Nurse vs. booklet & 108 & $£ 895$ & -0.0036 & Dominated \\
\hline Consultant vs. booklet & 98 & $E I 102$ & 0.0044 & $€ 250,455$ \\
\hline Consultant vs. nurse & 112 & $£ 207$ & 0.0076 & $£ 25,875$ \\
\hline $\begin{array}{l}\text { Cost effectiveness } \\
\text { analysis }\end{array}$ & $\mathbf{N}$ & Incremental cost $(t)$ & $\begin{array}{l}\text { Incremental mean change } \\
\text { in IBDQ-B score }\end{array}$ & $\begin{array}{l}\text { ICER (incremental cost per } \\
\text { 6-point IBDQ-B score gain) }\end{array}$ \\
\hline Nurse vs. booklet & 123 & $€ 878$ & 4.12 & $£ \mid 279$ \\
\hline Consultant vs. booklet & 116 & $£ 1076$ & 5.07 & $£ \mid 273$ \\
\hline Consultant vs. nurse & 125 & $£ 198$ & 0.95 & $£|25|$ \\
\hline
\end{tabular}

Abbreviations: IBDQ-B, Inflammatory Bowel Disease Questionnaire; ICER, incremental cost-effectiveness ratio; QALY, quality-adjusted life year.

a WTP of $\sim £ 250,000$ per QALY gained, the consultant-led treatment is favored with $\sim 52 \%$ probability of being the most cost-effective treatment option (Figure 1). This result suggests that although the consultant-led treatment leads to marginally better treatment outcomes on average, than either usual care (booklet) or the nurse-led treatment, the gain in QALY is too small to offset the relatively high incremental cost.

\section{Cost-effectiveness analysis}

Results of the probabilistic analysis using the IBDQ-B change from baseline differ from those using the QALY as the outcome measure (Table 3; Figure 2). For the IBDQ-B analysis, the bootstrap samples, for both the comparison of the consultant and the nurse with the booklet arm, are to the right of the zero line indicating both achieved a benefit compared to the usual care/booklet arm.

The CEAF suggests that at lower willingness to pay per 6-point gain in IBDQ-B score, the booklet has the highest probability of being the most cost-effective intervention. At $\sim £ 1280$, this shifts in favor of the nurse-led arm, which has $\sim 45 \%$ probability of being the most cost-effective intervention. Beyond a willingness to pay of $£ 2000$ per 6-point IBDQ-B change, the consultant-led intervention is favored at a probability of $\sim 50 \%$. This probability tends asymptotically towards a probability of $\sim 62 \%$ as the willingness to pay increases to $£ 8000$. This shift towards the consultant-led intervention is driven by the marginally better health outcomes this intervention achieved. However, without a defined threshold willingness to pay per 6-point change in IBDQ-B score, which is independently justifiable (equivalent to the NICE $£ 30,000$ per QALY), it is hard to make a judgment over which intervention is most favored by this analysis. Both the nurse- and consultant-led intervention achieve better health outcomes, as measured using the IBDQ-B; however, on average neither have a high likelihood of being deemed the most cost-effective, when compared to the booklet arm alone. 
Jordan et al

Dovepress
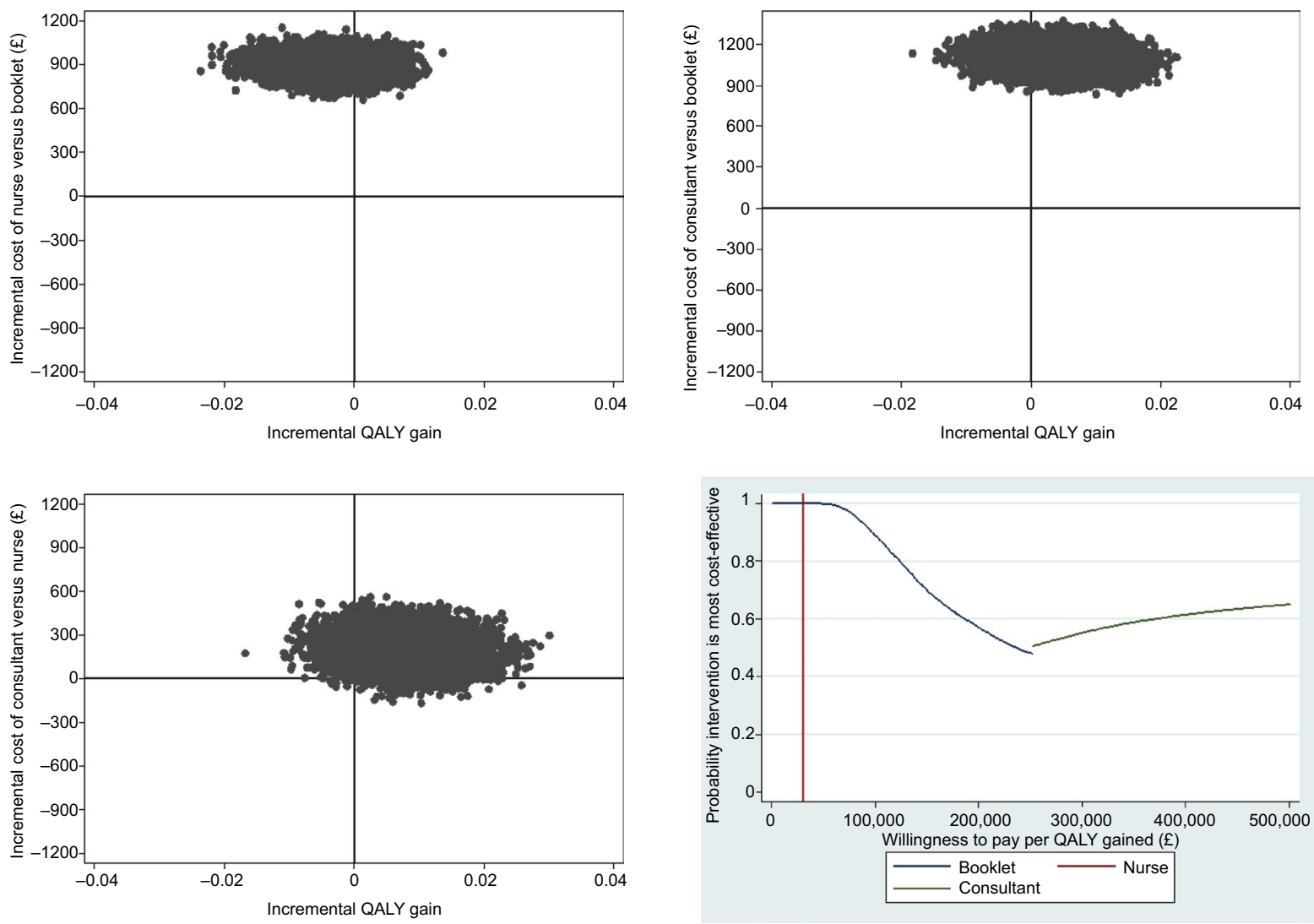

Figure I QALY scores: cost-effectiveness planes and cost-effectiveness acceptability frontier. Abbreviation: QALY, quality-adjusted life years.
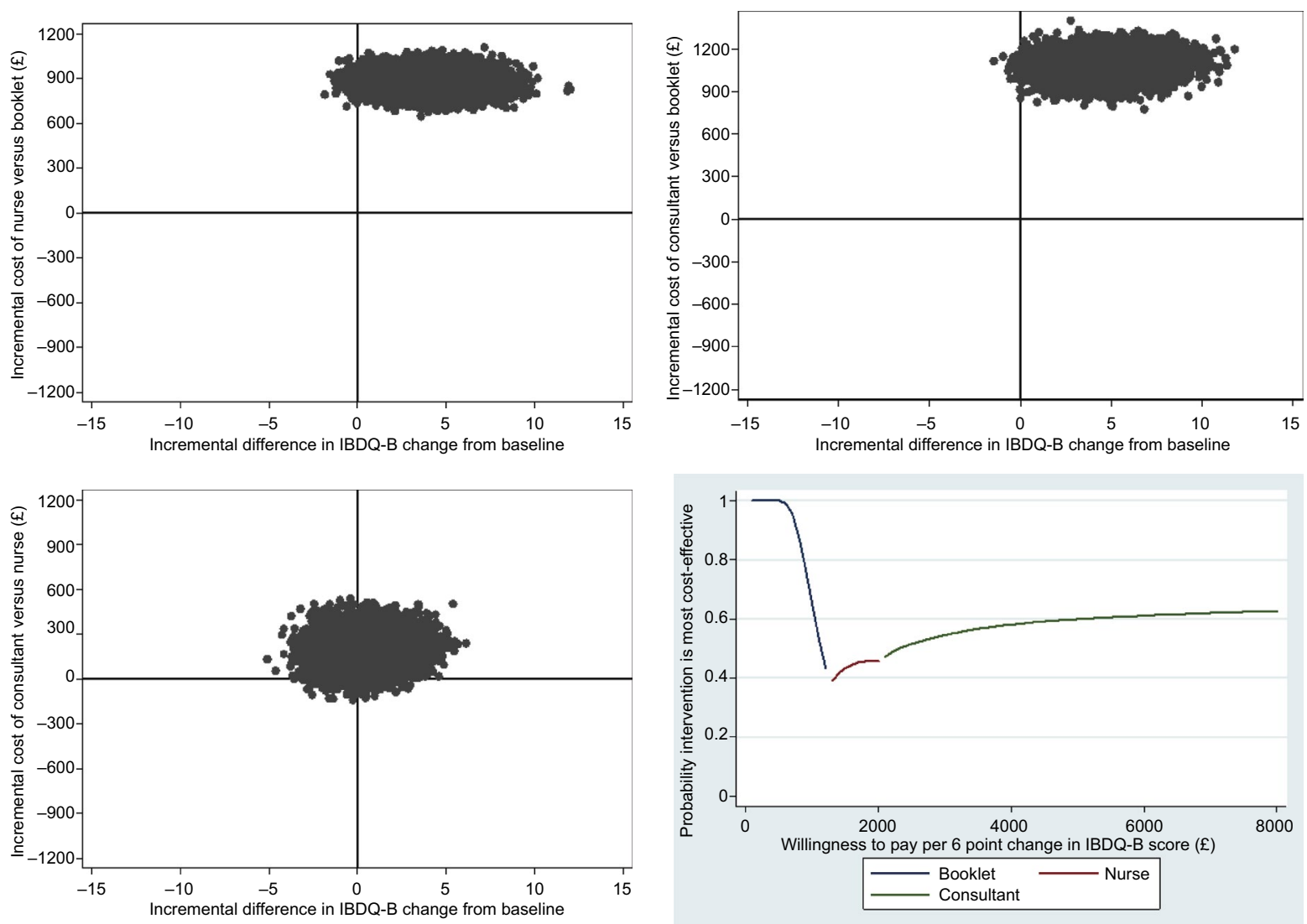

Figure 2 IBDQ-B scores: cost-effectiveness planes and cost-effectiveness acceptability frontier. Abbreviation: IBDQ-B, inflammatory bowel disease questionnaire.

246

submit your manuscript | www.dovepress.com

ClinicoEconomics and Outcomes Research 2017:9

Dovepress 


\section{Discussion}

The provision of algorithmic-based treatment to patients experiencing enduring gastrointestinal symptoms after radiotherapy for cancer costs $\sim £ 1100$ when delivered by a consultant gastroenterologist and $£ 895$ when delivered by a specialist nurse (British pounds 2013). Symptoms improved to a similar extent in both algorithmic care groups over a 6-month period, and significantly more than in a group of patients receiving only a self-help booklet. At a societal willingness to pay below $£ 1280$ for a clinically significant improvement in symptoms (i.e., at least a 6-point change in IBDQ-B), the booklet has a high probability of being the most cost-effective option. As the willingness to pay rises above that level, the nurse and then the consultant become more likely to be cost-effective, but the probabilities do not rise above $50 \%$ until a willingness to pay of about $£ 3000$ is reached. Simply put, the algorithmic care achieves better outcomes as reflected in IBDQ-B scores, and this comes at an additional cost of around $£ 1000$.

The finding that outcomes of nursing care are largely equivalent to those of doctors is consistent with other studies as shown in endoscopy clinics ${ }^{26}$ and in soft tissue clinics in an emergency department. ${ }^{27}$ As well as having similar outcomes, the use of nurses has also been shown to reduce costs compared to doctors in outpatient departments ${ }^{28}$ and rheumatoid arthritis clinics. ${ }^{29}$ As other studies compare the two types of practitioners in the delivery of usual care, this study focused on the evaluation of a new intervention. Lower costs associated with the nurse-led care was largely attributable to salary differences, and less medication prescribing; the application of the algorithm resulted in very similar diagnostic and non-pharmacological treatment costs for the two types of practitioners.

Although patients in the trial recorded clinically and statistically significant improvements in bowel symptoms as a result of the nurse and consultant interventions, commensurate changes in generic health-related quality of life (QALYs) were not found. At the upper limit of the NICE threshold of willingness to pay of $£ 30,000 / \mathrm{QALY}$ gained, the booklet arm has a near $100 \%$ chance of being the most cost-effective option. The incremental cost of consultant- and nurse-led treatments, compared to usual care, were too high, given the small utility gains, to be deemed cost-effective options. A societal willingness to pay in the region of $£ 250,000$ /QALY gained is required before the consultant treatment achieves a $50 \%$ probability of being the most cost-effective option. The nurse option was not found to be cost-effective compared to either the booklet- or consultant-led care. A comparison of care of people with rheumatoid arthritis by nurse and rheumatologist similarly showed the nurse was not cost-effective, as measured using QALYs estimated from generic utility scores. ${ }^{29}$ Therefore, caution must be taken when considering provision of care based solely on the generic health-related quality of life instruments that may not capture the full patient benefit of symptom-specific interventions. The marginal cost of treating post-radiotherapy symptoms identified in this study $(\sim £ 1000)$ is relatively small when considering the overall cost of the cancer treatment already incurred for this patient group. Moreover, inflammatory bowel disease, which affects equivalent numbers of people and incurs similar symptoms, is routinely managed through specialist supportive secondary care. ${ }^{30}$

The economic analysis had several limitations. Bias may have arisen from non-random missing data. The conversion of SF-12 scores to SF-6 and then EQ-5D utility may have caused lack of precision in the calculation of QALYs. In addition, given a significant change was detected in the disease-specific IBDQ-B measure, it is possible that the SF-12 instrument may have been insensitive to changes in the symptom-specific quality of life of these patients. Although attempts were made to gather data from participants on use of other services over the 6-month observation period, many did not return usable information so potential healthcare savings to offset the cost of the intervention could not be explored. The length of follow-up consultations was not recorded, and the assumption of 15 minutes may underestimate the costs. The usual care comparison condition was not entirely representative of treatment as usual as many people with gastrointestinal symptoms after radiotherapy do not receive any information or treatment in the UK. Hence, provision of a booklet and the opportunity to receive treatment from the consultant if symptoms remained at 6 months represented an enhancement on usual care for people recruited to the trial.

\section{Conclusion}

The clinical trial and cost-effectiveness analysis were conducted robustly and evaluated an important new treatment for cancer survivors. This patient group often tolerates significant gastrointestinal symptoms and reduced quality of life in the belief that this is the normal sequel to radiation therapy. Although evidence for cost-effectiveness according to the NICE criteria was not found in this study, participants in both intervention groups recorded, on average, significantly (statistically and clinically) greater improvement in bowel symptoms than those in the booklet-only group. Monitoring the continuing side effects of radiation therapy and providing treatment for radiation injury would add some $£ 1000$ to the overall package of care for cancer patients which, for example, currently costs between $£ 10,000$ and $£ 15,000$ for bowel cancer (based 
on applying the GDP deflator to figures from 2007). ${ }^{31,32}$ The failure of the economic evaluation to show cost-effectiveness of the treatment algorithm in terms of cost per QALY gained may reflect the reliance of the criterion on a generic measure of health-related quality of life and should not be viewed in isolation. Improvements in this instance are highly symptom specific; therefore, relying on the generic measure of quality of life may not capture the full patient benefit from this intervention, as evidenced by the clinical IBDQ-B results. ${ }^{5}$ The cost-effectiveness of the booklet suggests that a care pathway might be developed where patients with bowel symptoms after pelvic radiotherapy are offered the self-help booklet first, and if troublesome symptoms remain after 6 months, they are referred for specialist algorithmic care.

\section{Acknowledgments}

This research was funded by the National Institute for Health Research (NIHR) under its Research for Patient Benefit (RfPB) Programme (grant number PB-PG-0706-10175). The views expressed are those of the authors and not necessarily those of the NHS, the NIHR or the UK Department of Health.

We thank the patients in the trial steering group, James Lindsay, Albert Fisher, Joan Grainger, and Lisl Klein for insightful and constructive input as the initial protocol was developed and at the 3-monthly trial steering group meetings as the study progressed. We thank Kabir Mohammed, Kiell Pennert and Karen Thomas for statistical input into the study design and analysis of the clinical data. We also thank Julie Duncan, Robyn Reagon, Foteini Silia, and Ruth Stafferton for help with patient recruitment and management at different stages of the study and Caragh Flannery for help with the economic analysis.

\section{Disclosure}

The authors report no conflicts of interest in this work.

\section{References}

1. Andreyev HJN, Gastrointestinal problems following radiotherapy. Clin Oncol. 2007;19(10):790-799.

2. Andreyev HJN, Wotherspoon A, Denham JD, Hauer-Jensen M. Defining pelvic-radiation disease for the survivorship era. Lancet Oncol. 2010;11(4):310-312.

3. Department of Health. National Cancer Survivorship Initiative Vision. Available from: http://www.ncsi.org.uk/wp-content/uploads/NCSIVision-Document.pdf. Accessed April 5, 2015.

4. Deham J, Hauer-Jensen M. Radiation induced bowel injury: a neglected problem. Lancet. 2013;382(9910): 2016-2017.

5. Andreyev HJN, Benton B, Lalji A, et al. Algorithm-based management of patients with gastrointestinal symptoms in patients after pelvic radiation treatment (ORBIT): a randomised controlled trial. Lancet. 2013;382(9910):2084-2092).
6. Guyatt G, Mitchell A, Irvine E, et al. A new measure of health status for clinical trials in inflammatory bowel disease. Gastroenterology. 1989;96(3):804-810.

7. Khalid U, McGough C, Hackett C, et al. A modified inflammatory bowel disease questionnaire and the Vaisey incontinence questionnaire are more sensitive measures of acute gastrointestinal toxicity during pelvic radiotherapy than RTOG grading. Int J Radiat Oncol Biol Phys. 2006;64(5):1432-1441.

8. Gami B, Harrington K, Blake P, et al. How patients manage gastrointestinal symptoms after pelvic radiotherapy. Aliment Pharmacol Ther. 2003;18:987-994.

9. Olopade FO, Norman AR, Blake P, et al. The inflammatory bowel disease questionnaire and the Vaizey Incontinence Questionnaire are useful to identify gastrointestinal toxicity after pelvic radiotherapy. $\mathrm{Br}$ J Cancer. 2005;92:1663-1670.

10. Gillespie C, Cooke C, Hackett C, Andreyev HJN. The clinical needs of patients with chronic gastrointestinal symptoms after pelvic radiotherapy. Aliment Pharmacol Ther. 2007;26(4):555-563.

11. Benton BE, Norton C, Lindsay JO, Dolan S, Andreyev HJN. Can nurses manage gastrointestinal symptoms arising from pelvic radiation disease? Clin Oncol. 2011;23(8):538-551.

12. Andreyev HJN, Davidson SE, Gillespie C, Allum WH, Swarbrick E. Practice guidance on the management of acute and chronic gastrointestinal problems arising as a result treatment for cancer. Gut. 2012; 61(2):179-192.

13. Andreyev HJN, Muls A, Norton C, et al. Guidance: the practical management of gastrointestinal symptoms of Pelvic Radiation Disease. Frontline Gastroenterol. 2014;6(1):53-72.

14. Phillips C. What is a QALY? Available from: http://www.medicine. ox.ac.uk/bandolier/painres/download/whatis/qaly.pdf. Accessed April 5, 2015.

15. McCabe C1, Claxton K, Culyer AJ. The NICE cost-effectiveness threshold: what it is and what that means. Pharmacoeconomics. 2008; 26(9):733-744.

16. Ware JJ, Kosinski M, Keller SD. A 12-item short form health survey: construction of scales and preliminary tests of reliability and validity. Medical Care. 1996;34(3):220-233.

17. Brazier J, Roberts J. The estimation of a preference-based measure of health from SF-12. Med Care. 2004;42(9):851-859.

18. EuroQoL, What is EQ-5D? Available from: http://www.euroqol.org. Accessed April 5, 2015.

19. Gray A, Rivero-Arias O, Clarke P. Estimating the associations between SF-12 responses and EQ-5D utility values by response mapping. Med Decis Making. 2006;26(1):18-29.

20. Dolan P, Gudex C, Kind P, Williams A. A social tariff for EuroQoL: results from a UK general population survey. University of York, Centre for Health Economics, Discussion Paper 138, 1995. Available from: http://www.york.ac.uk/che/pdf/DP138.pdf. Accessed April 5, 2015.

21. Hlavaty T, Persoons P, Vermiere S, et al. Evaluation of short term responsiveness and cutoff values of inflammatory bowel disease questionnaire in Crohn's disease. Inflamm Bowel Dis. 2006;12(3):199-204.

22. Department of Health. National Reference Costs 2013. Available from: https://www.gov.uk/government/publications/nhs-reference-costs2013-to-2014. Accessed March 6, 2017.

23. Curtis L. Unit costs of health and social care 2013, Available from: http://www.pssru.ac.uk. Accessed April 5, 2015.

24. Manca A, Hawkins N, Sculpher MJ. Estimating mean QALYs in trialbased cost-effectiveness analysis: the importance of controlling for baseline utility. Health Econ. 2005;14(5):487-496.

25. Briggs AH, Wonderling DE, Mooney CZ. Pulling cost-effectiveness analysis up by its bootstraps: a non-parametric approach to confidence interval estimation. Health Econ. 1997;6(4):327-340.

26. Williams J, Russell I, Durai D, et al. Effectiveness of nurse delivered endoscopy: findings from randomised multi-institution nurse endoscopy trial (MINuET). BMJ. 2009;338:b231. 
27. Middleton McClellan C, Cramp F, Powell J, Benger J. A randomised trial comparing the clinical effectiveness of different emergency department healthcare professionals in soft tissue injury management. BMJ Open. 2012;2(6):e001092.

28. Kirkpatrick K, Kaasalainen S, Donald F, et al. The effectiveness and cost-effectiveness of clinical nurse specialists in outpatient roles: a systematic review. J Eval Clin Pract. 2014;20(6):1106-1123.

29. Ndosi M, Lewis M, Hale C, et al. The outcome and cost-effectiveness of nurse-led care in people with rheumatoid arthritis: a multicentre randomised controlled trial. Ann Rheum Dis. 2014;73(11):1975-1982.
30. Andreyev HJN. GI consequences of cancer treatment: a clinical perspective. Radiat Res. 2016;185(4):341-348.

31. York Health Economics Consortium, University of Sheffield. Bowel cancer services: costs and benefits. Summary report to the Department of Health. April 2007. Available from: https://www.sheffield.ac.uk/ polopoly_fs/1.44049!/file/FinalBowelCancerSummaryReport-Apr07. pdf. Accessed March 10, 2016.

32. Charlesworth A. Why is healthcare inflation greater than general inflation? J Health Serv Res Policy. 2014;19(3):129-130.
ClinicoEconomics and Outcomes Research

\section{Publish your work in this journal}

ClinicoEconomics and Outcomes Research is an international, peerreviewed open-access journal focusing on health technology assessment, pharmacoeconomics and outcomes research in the areas of diagnosis, medical devices, and clinical, surgical and pharmacological intervention. The economic impact of health policy and health systems
Submit your manuscript here: https://www.dovepress.com/clinicoeconomics-and-outcomes-research-journal

organization also constitute important areas of coverage. The manuscript management system is completely online and includes a very quick and fair peer-review system, which is all easy to use. Visit http://www.dovepress.com/testimonials.php to read real quotes from published authors. 